PROCEEDINGS OF THE

AMERICAN MATHEMATICAL SOCIETY

Volume 140, Number 3, March 2012, Pages 999-1010

S 0002-9939(2011)11307-8

Article electronically published on September 1,2011

\title{
INNER TUBE FORMULAS FOR POLYTOPES
}

\author{
ŞAHIN KOÇAK AND ANDREI V. RATIU
}

(Communicated by Ken Ono)

\begin{abstract}
We show that the volume of the inner $r$-neighborhood of a polytope in the $d$-dimensional Euclidean space is a pluriphase Steiner-like function, i.e. a continuous piecewise polynomial function of degree $d$, thus proving a conjecture of Lapidus and Pearse. We discuss also the degree of differentiability of this function and give a lower bound in terms of the set of normal vectors of the hyperplanes defining the polytope. We also give sufficient conditions for the highest differentiability degree to be attained.
\end{abstract}

\section{INTRODUCTION}

The Steiner formula is a beautiful cornerstone of convex geometry dating from 1840. It states that the volume of the $r$-parallel set of a given convex and compact set in $\mathbb{R}^{d}$ can be expressed as a polynomial function of $r$ of degree $d$ (for $r \geq 0$ ) ([1] Proposition 12.3.6, 5] Formula 4.1.1). The $r$-parallel set $A_{r}$ of a set $A \subset \mathbb{R}^{d}$ is by definition the Minkowski sum of $A$ with the ball of radius $r$ in $\mathbb{R}^{d}$, or, in other words, the set of points in $\mathbb{R}^{d}$ with distance at most $r$ to $A$. As a simple example, the following formula holds for a convex, compact set $A$ in the plane, having non-empty interior:

$$
\operatorname{area}\left(A_{r}\right)=\operatorname{area}(A)+\operatorname{length}(\partial A) r+\pi r^{2} \quad\left(A \subset \mathbb{R}^{2}\right) .
$$

Figure 1 gives a "proof without words" for a convex polygon in the plane. In

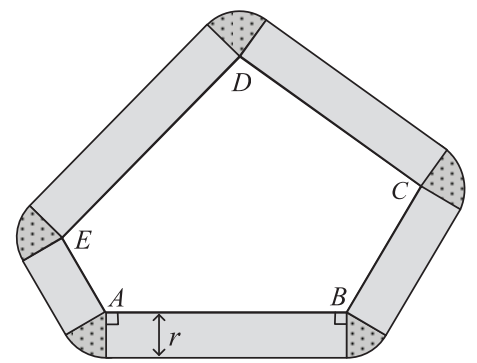

Figure 1. The outer $r$-neighborhood of a convex polygon in the plane. Note that the sector-volumes add up to $\pi r^{2}$.

Received by the editors December 8, 2010.

2010 Mathematics Subject Classification. Primary 52B11; Secondary 52A38.

Key words and phrases. Polytope, Steiner formula, tube formula.

(C)2011 American Mathematical Society Reverts to public domain 28 years from publication 
space, we have the following formula for a convex body $A$, with smooth boundary $\partial A$ :

$$
\operatorname{vol}\left(A_{r}\right)=\operatorname{vol}(A)+\operatorname{area}(\partial A) r+H(\partial A) r^{2}+\frac{4}{3} \pi r^{3},
$$

where $H(\partial A)$ denotes the total mean curvature of $(\partial A)$ (2] Theorem 51, 4] Theorem 10.2).

In 1939, H. Weyl proved that volumes of tubular $r$-neighborhoods of smooth submanifolds of $\mathbb{R}^{d}$ can be expressed as polynomials of $r$, for $r$ small enough) and gave impressive explicit formulas for the coefficients of the polynomial in terms of the curvature tensor of the submanifold. As simple but interesting examples we note two cases. For a smoothly embedded circle $\mathrm{C}$ in $\mathbb{R}^{d}$ it holds that

$$
\operatorname{vol}\left(C_{r}\right)=\alpha_{d-1} \operatorname{length}(C) r^{d-1},
$$

where $\alpha_{d-1}$ denotes the volume of the $(d-1)$-dimensional unit ball.

For a closed orientable surface $S$ in $\mathbb{R}^{d}$ one has the following formula:

$$
\operatorname{vol}\left(S_{r}\right)=\alpha_{d-2} \text { area }(S) r^{d-2}+\alpha_{d} \chi(S) r^{d},
$$

where $\chi(S)$ is the Euler characteristic of $S$ (1 12.10.9.2).

Now, convex sets need not be smooth and submanifolds need not be convex. In 1959 Federer created the notion of "sets of positive reach", including both of these important classes of sets, the convex sets and $\left(\mathcal{C}^{2}\right.$-smooth) submanifolds in $\mathbb{R}^{d}$ of any codimension. A closed set $A \subset \mathbb{R}^{d}$ is called of positive reach, if there exists a parallel set $A_{r}$ (with $r>0$ ) such that any point of $A_{r}$ has a unique nearest point in $A$. The supremum of these $r$ is called the reach of $A$. Federer proved that Steiner formula still holds for this larger class of sets, with $r$ within the reach of the set ([3] Theorem 5.6).

It seems that during the long history of this problem, the inner $r$-neighborhoods of convex bodies (or of closed hypersurfaces), i.e. the set of all points at distance at least $r>0$ away from the exterior, was not an issue of interest. From the above formulas one sees that the area of the inner $r$-neighborhood of a convex set $A$ with smooth boundary in $\mathbb{R}^{2}$ can be expressed (for small $r$ ) as length $(\partial A) r-\pi r^{2}$ and the volume of the inner $r$-neighborhood of a convex body with smooth boundary in $\mathbb{R}^{3}$ as area $(\partial A) r-H(\partial A) r^{2}+\frac{4}{3} \pi r^{3}$.

If the boundary of a domain is not $\mathcal{C}^{2}$-differentiable or if it is not of positive reach from inside, then there are, to our knowledge, no available Steiner-like formulas. In the negative, for many simple convex domains (for example the semidisc in the plane; see Example 2) the volume of the inner $r$-neighborhood is definitely nonpolynomial even for small positive $r$.

The interest in volumes of inner neighborhoods of domains in $\mathbb{R}^{d}$ was actualized by the recent research of Lapidus and coworkers, notably by Lapidus and Pearse, who established a startling formula for volumes of neighborhoods of fractals, whereby they defined new complex dimensions for fractals, related the neighborhoods of fractals to inner neighborhoods of some associated sequences of open domains, and expressed the volume of the $r$-neighborhood of a fractal as a sum of residues of a certain associated $\zeta$-function at the complex dimensions of the fractal (6] Theorem 8.1, 7] Theorem 7.4, 9] Theorem 5.7, [10] Theorem 1).

In this article we consider simple but important types of convex bodies, the polytopes in $\mathbb{R}^{d}$. The inner neighborhoods stabilize at the inradius. We prove a conjecture of Lapidus and Pearse ( 8 , Conjecture 1$)$ stating that the volume of the 


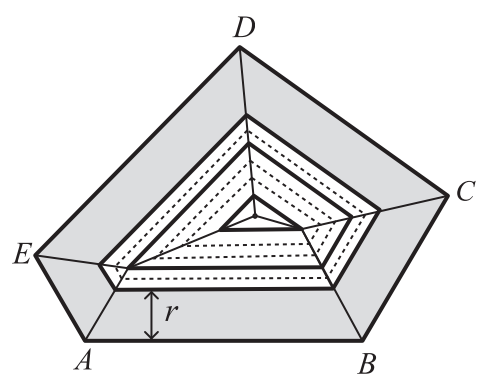

Figure 2. The inner $r$-neighborhood of a convex polygon $P$ in the plane. For small $r$, until the passage to a quadrangle, its area is given by the formula $A(r)=\operatorname{perimeter}(P) \cdot r-\left(\sum \tan \left(\alpha_{i} / 2\right)\right) \cdot r^{2}$, where the $\alpha_{i}$ 's are the outer angles of the polygon.

inner $r$-neighborhood of a (convex, compact) polytope is a continuous piecewise polynomial function for $r \geq 0$. We call such a behavior pluriphase Steiner-like. Figure 2 shows a quatre-phase Steiner-like example in the plane.

We discuss also the degree of differentiability of this function and give a lower bound in terms of the set of normal vectors of the hyperplanes defining the polytope. We give also sufficient conditions for the highest differentiability degree $d-1$ to be attained. There are interesting consequences resulting from these considerations about the geometric $\zeta$-function for polytopes in the sense of Lapidus and Pearse, but we want to restrict ourselves in this article to the convex geometry framework.

\section{The Volume FUnCtion}

Let $P$ be a convex body in $\mathbb{E}^{d}$, i.e. a compact convex subset with non-empty interior. For all positive $r$ we denote by $P(r)$ the $r$-interior of $P$,

$$
P(r)=\{Q \in P \mid \mathrm{d}(Q, \partial P) \geq r\},
$$

and we call the set $P \backslash P(r)$ the inner $r$-neighborhood of $\partial P$. The aim of this article is the study of the inner neighborhood volume function

$$
V_{P}(r)=\operatorname{vol}_{d}(P \backslash P(r))
$$

or, equivalently, of the volume function

$$
W_{P}(r)=\operatorname{vol}_{d}(P(r))=\operatorname{vol}_{d}(P)-V_{P}(r) .
$$

We start with a straightforward example.

Example 1. If $P$ is a $d$-dimensional Euclidean ball of radius $g$, then

$$
V_{P}(r)= \begin{cases}\sum_{i=0}^{d-1} \kappa_{i}(P) r^{d-i}, & \text { if } r \leq g, \\ \operatorname{vol}_{d}(P), & \text { if } r \geq g .\end{cases}
$$

Here the coefficients are

$$
\kappa_{i}(B)=(-1)^{d-i-1}\left(\begin{array}{c}
d \\
i
\end{array}\right) \frac{\operatorname{vol}_{d}(B)}{g^{d-i}}, \quad \text { for all } i .
$$

The inradius of a convex body $P$ is defined as the maximum positive value $g$ such that $P$ contains a ball of radius $g$. It is then clear that the volume function $W_{P}$ stabilizes at $g$, i.e. $W_{P}(r)=0$, for all $r \geq g$. 
Let $d$ be a non-negative integer.

Definition 1. A degree d pluriphase Steiner-like function $\varphi$ is a continuous function

$$
\varphi:[0,+\infty) \longrightarrow[0,+\infty)
$$

such that there is a partition of the non-negative half axis into $m$ intervals $0=g_{0}<$ $g_{1}<\cdots<g_{m}<+\infty$ such that

- for each $1 \leq i \leq m$, the restriction of $\varphi$ on the subinterval $\left[g_{i-1}, g_{i}\right]$ is a degree $d$ polynomial

$$
\varphi(r)=\sum_{k=0}^{d} \kappa_{d-k, i} r^{k}, \quad \text { for all } r \in\left[g_{i-1}, g_{i}\right]
$$

and

- $\varphi$ is constant on $\left[g_{m},+\infty\right)$.

The $g$-value of $\varphi$ is by definition $g_{m}$. The class of pluriphase Steiner-like functions is clearly closed under taking linear combinations and under integration. In case $m=1, \varphi$ is called a monophase Steiner-like function and the coefficient $\kappa_{d-k, 1}$ of $r^{k}$ is denoted simply by $\kappa_{d-k}$.

The main result of this article is to show that whenever $P$ is a polytope in $\mathbb{E}^{d}$, i.e. a convex body that is the intersection of finitely many closed half-spaces in $\mathbb{E}^{d}$, the inner neighborhood volume function $V_{P}$ is a degree $d$ pluriphase Steiner-like function. If $\left\{\mathcal{H}_{1}, \mathcal{H}_{2}, \ldots, \mathcal{H}_{m}\right\}$ is a minimal collection of half-spaces in $\mathbb{E}^{d}$ defining $P$, the sets $F_{j}=P \cap \partial \mathcal{H}_{j}$ are called the facets of $P$. Their union equals the boundary of the polytope, and the total surface area of the polytope is

$$
\operatorname{vol}_{d-1}(\partial P)=\sum_{j=1}^{m} \operatorname{vol}_{d-1}\left(F_{j}\right) .
$$

Note that for $r$ small enough, $W_{P}(0)-W_{P}(r)=\frac{r}{2}\left(\operatorname{vol}_{d-1}(\partial P)+\operatorname{vol}_{d-1}(\partial P(r))\right)$. We will use the resulting differentiation formula for the volume function:

$$
W_{P}^{\prime}(0)=-\sum_{j=1}^{m} \operatorname{vol}_{d-1}\left(F_{j}\right),
$$

valid for all $d$-dimensional polytopes $P$ with facets $F_{1}, F_{2}, \ldots, F_{m}$. As each facet $F_{j}$ is a $(d-1)$-dimensional polytope, the proof will essentially consist of an inductive argument on dimension. By definition, a 0-dimensional polytope is a point and its 0 -dimensional volume is simply 1 .

By contrast, even for very simple convex subsets in the plane the inner neighborhood volume function is not necessarily a Steiner-like function, as the following example shows.

Example 2. Let $P$ be the semidisc $\left\{(x, y) \in R^{2} \mid 0 \leq y \leq \sqrt{1-x^{2}}\right\}$. Then the $r$-interior of $P$ is

$$
P(r)=\left\{(x, y) \in R^{2} \mid r \leq y \leq \sqrt{(1-r)^{2}-x^{2}}\right\}
$$

and

$$
V_{P}(r)= \begin{cases}\frac{\pi}{2}-(1-r)^{2} \arccos \left(\frac{r}{1-r}\right)+r \sqrt{1-2 r}, & \text { if } r \leq \frac{1}{2} \\ \frac{\pi}{2}, & \text { if } r \geq \frac{1}{2}\end{cases}
$$


Note that $V_{P}$ is of differentiability class $\mathcal{C}^{1}$ on $[0,+\infty)$, but not $\mathcal{C}^{2}$, as

$$
\lim _{r \nearrow \frac{1}{2}} V_{P}^{\prime \prime}(r)=-\infty
$$

The differentiability class of the volume function reflects the metric properties of the polytope. For instance among all the rectangles, only the cubes have volume function of maximum differentiability class, as the following example proves:

Example 3. Let $a_{1} \leq a_{2} \leq \cdots \leq a_{d}$ and let $R$ be the $d$-dimensional rectangle

$$
R=R_{a_{1}, a_{2}, \ldots, a_{d}}=\left\{x=\left(x_{1}, x_{2}, \ldots, x_{d}\right)|| x_{i} \mid \leq a_{i}, \text { for all } i\right\}
$$

of inradius $g=a_{1}$. Then $V_{R}$ is a monophase Steiner-like function

$$
V_{R}(r)= \begin{cases}2^{d} a_{1} a_{2} \cdots a_{d}-2^{d}\left(a_{1}-r\right)\left(a_{2}-r\right) \cdots\left(a_{d}-r\right), & \text { if } r \leq g, \\ 2^{d} a_{1} a_{2} \cdots a_{d}, & \text { if } r \geq g .\end{cases}
$$

Thus the coefficients are

$$
\kappa_{d-k}=(-1)^{k-1} \operatorname{vol}_{d-k}\left(R_{(d-k)}\right), \quad \text { for } 1 \leq k \leq d .
$$

Here $\operatorname{vol}_{d-k}\left(R_{(d-k)}\right)$ denotes the sum of the appropriate volumes of all the $(d-k)$-dimensional faces of the polytope $R$ and $\kappa_{0}=(-1)^{d-1} \sharp\left(R_{(0)}\right)$. Note also the important fact that, if $m=\sharp\left\{1 \leq i \leq d \mid a_{i}=g\right\}$, then $V_{R}$ is of differentiability class $\mathcal{C}^{(m-1)}$, but not $\mathcal{C}^{m}$. Indeed, $g$ is a root of the polynomial $2^{d}\left(a_{1}-r\right)\left(a_{2}-r\right) \cdots\left(a_{d}-r\right)$ of multiplicity exactly $m$.

The following theorem characterizes the polytopes in $\mathbb{E}^{d}$ for which the function $V_{P}$ is a monophase Steiner-like function of maximal differentiability class, i.e. of class $\mathcal{C}^{(d-1)}$.

Theorem 1. A necessary and sufficient condition for the inner neighborhood volume function $V_{P}$ of a d-dimensional polytope $P$ to be a monophase Steiner-like function of class $\mathcal{C}^{(d-1)}$ on $[0,+\infty)$ is that $P$ admits an inscribed Euclidean $d$ dimensional ball. Moreover, in this case the coefficients $\kappa_{i}(P)$ of $P$ satisfy

$$
\kappa_{i}(P)=(-1)^{d-i-1}\left(\begin{array}{l}
d \\
i
\end{array}\right) \frac{\operatorname{vol}_{d}(P)}{g^{d-i}},
$$

for all $i=0,1, \ldots, d-1$.

Proof. We first prove the sufficiency of the condition. Assume that the origin $O$ is the center of a $d$-dimensional ball of radius $g$ inscribed in $P$, i.e. tangent to all the facets of $P$. For each $r$ in $[0, g]$ let $\tilde{P}(r)=\left(1-\frac{r}{g}\right) P$. Note that, due to the assumption above, the polytopes $\tilde{P}(r)$ and $P(r)$ coincide, for all $r$. Thus for each $r$ in $[0, g]$,

$$
\begin{aligned}
V_{P}(r) & =\operatorname{vol}_{d}(P \backslash \tilde{P}(r))=\operatorname{vol}_{d}(P)\left(1-\left(1-\frac{r}{g}\right)^{d}\right) \\
& =\operatorname{vol}_{d}(P) \sum_{i=0}^{d-1}(-1)^{d-i-1}\left(\begin{array}{c}
d \\
i
\end{array}\right)\left(\begin{array}{c}
r \\
g
\end{array}\right)^{d-i},
\end{aligned}
$$

and for $r>g, V_{P}(r)=\operatorname{vol}_{d}(P)$.

In order to prove the necessity, note that for any $d$-dimensional polytope $P$, the inradius $g$ satisfies

$$
g=\min \left\{r>0 \mid \operatorname{vol}_{d}(P(r))=0\right\}=\min \left\{r>0 \mid V_{P}(r)=\operatorname{vol}_{d}(P)\right\} .
$$


Assume now that $V_{P}$ is a monophase Steiner-like function. Then its $g$-value equals the inradius of $P$. Writing down the continuity conditions at $r=g$ for the function

$$
V_{P}(r)=\left\{\begin{array}{cc}
\sum_{i=0}^{d-1} \kappa_{i}(P) r^{d-i} & \text { if } r<g \\
\operatorname{vol}_{d}(P) & \text { if } r \geq g
\end{array}\right.
$$

and for its first $(d-1)$ derivatives, we obtain the non-singular triangular system of linear equations in the $d$ coefficients $\kappa_{i}$, with $0 \leq i<d$,

$$
\sum_{i=0}^{d-j}\left(\begin{array}{c}
d-i \\
j
\end{array}\right) \kappa_{i}(P) g^{d-i-j}=0, \quad \text { for all } j=0, \ldots, d-1,
$$

where, for simplicity we have denoted the constant $\operatorname{vol}_{d}(P)$ by $-\kappa_{d}(P)$. The unique solution of this system is the one stated in the theorem, as can be directly verified.

Consider that the ball of radius $g$ centered at the origin is contained in $P$. Then $\left(1-\frac{r}{g}\right) P \subset P(r)$. Since

$$
\begin{aligned}
V_{P}(r) & =\sum_{i=0}^{d-1} \kappa_{i}(P) r^{d-i} \\
& =\operatorname{vol}_{d}(P) \sum_{i=0}^{d-1}(-1)^{d-i-1}\left(\begin{array}{c}
d \\
i
\end{array}\right)\left(\frac{r}{g}\right)^{d-i} \\
& =\operatorname{vol}_{d}(P)\left(1-\left(1-\frac{r}{g}\right)^{d}\right)=\operatorname{vol}_{d}\left(P \backslash\left(1-\frac{r}{g}\right) P\right),
\end{aligned}
$$

we conclude that $P \backslash P(r)=P \backslash\left(1-\frac{r}{g}\right) P$, for all $r<g$. Thus the distance of the origin to all the facets is exactly $g$, and the ball of radius $g$ considered is inscribed in $P$.

\section{THE MAIN RESUlT}

In this section we prove that the volume function of any polytope is a pluriphase Steiner-like function. For that we introduce the concept of a gliding arrangement of hyperplanes in a Euclidean space. The hyperplanes supporting the facets of the "shrinking" polytope $P(r)$, as $r$ increases, constitute the gliding arrangement of hyperplanes adapted to the polytope $P$. This concept will allow an inductive argument on the dimension of the polytope.

By a hyperplane in $\mathbb{E}^{d}$ we will understand an oriented hyperplane $H$, where the orientation is given by a choice of a unit normal vector $N$.

Definition 2. A $d$-arrangement of hyperplanes is a finite family $\mathcal{A}$ of (not necessarily distinct) hyperplanes $H_{1}, H_{2}, \ldots, H_{m}$ in a $d$-dimensional real affine Euclidean space $\mathbb{E}^{d}$.

Definition 3. The signed distance $\mathrm{d}\left(Q, H_{0}\right)$ from a point $Q$ in $\mathbb{E}^{d}$ to the oriented hyperplane $H_{0}$ with unit normal vector $N_{0}$ is by definition $\mathrm{d}\left(Q, H_{0}\right)=\left\langle\overline{Q_{0} Q}, N_{0}\right\rangle$, where $Q_{0}$ is any point in the hyperplane $H_{0}$. The cells $C_{\varepsilon}$ of the arrangement $\mathcal{A}$ are indexed by $\varepsilon=\left(\varepsilon_{1}, \varepsilon_{2}, \ldots, \varepsilon_{m}\right) \in\{ \pm 1\}^{m}$ and are defined as the set

$$
C_{\varepsilon}=\left\{Q \in \mathbb{E}^{d} \mid \varepsilon_{j} \mathrm{~d}\left(Q, H_{j}\right) \geq 0, \text { for all } j\right\} .
$$

By convention, we consider cells with different multi-indices as different, even when empty. So a $d$-arrangement of cardinality $m$ defines exactly $2^{m}$ cells in $\mathbb{E}^{d}$. 
Definition 4. We say that the family of vectors $\mathcal{N}=\left\{N_{1}, N_{2}, \ldots, N_{m}\right\}$ in $\mathbb{R}^{d}$ has absolute rank absrk $\mathcal{N}=k$ if (i) any $k$ elements of $\mathcal{N}$ are linearly independent, and (ii) there are $k+1$ vectors in the family that are linearly dependent. The rank (respectively the absolute rank) of a $d$-arrangements $\mathcal{A}$ is defined to be the rank (respectively, the absolute rank) of the family of the normal vectors $\mathcal{N}$ of its elements.

For instance, the family $\left\{ \pm \mathbf{e}_{1}, \pm \mathbf{e}_{2}, \ldots, \pm \mathbf{e}_{d}\right\}$ of unit vectors in $\mathbb{R}^{d}$ has absolute rank 1 , while its rank is $d$. In general, the chain of inequalities holds: $1 \leq$ absrk $\mathcal{N} \leq$ $\operatorname{rk} \mathcal{N} \leq \min \{m, d\}$, for all non-empty families $\mathcal{N}$. By convention, we consider the absolute rank of an empty family to be null.

Definition 5. Let $H_{0}$ be an oriented hyperplane in $\mathbb{E}^{d}$, with $d \geq 2$. The trace of the $d$-arrangement $\mathcal{A}$ on $H_{0}$ is the family $\tilde{\mathcal{A}}_{0}$ of all the proper intersections of $H_{0}$ with each of the hyperplanes in $\mathcal{A}$, each intersection being endowed with the normal vector $\tilde{N}_{j}=\frac{p r_{0}\left(N_{j}\right)}{\left|p r_{0}\left(N_{j}\right)\right|}$, where $\operatorname{pr}_{0}(v)=v-\left\langle v, N_{0}\right\rangle N_{0}$ is the projection on the linear hyperplane subtending $H_{0}$, i.e.

$$
\tilde{\mathcal{A}}_{0}=\left\{\left(H_{j} \cap H_{0}, \frac{p r_{0}\left(N_{j}\right)}{\left|p r_{0}\left(N_{j}\right)\right|}\right)\right\},
$$

where $1 \leq j \leq m$ is such that $H_{j} \cap H_{0}$ is a proper subspace in $H_{0}$.

Note that, for all $j$ such that $1 \leq j \leq m$, the trace $\tilde{\mathcal{A}}_{j}$ on the hyperplane $H_{j}$ is a (possibly empty) $(d-1)$-arrangement of absolute rank at least $k-1$ and of rank at least $\operatorname{rk} \mathcal{A}-1$. In particular, if $\operatorname{rk} \mathcal{A} \geq 2$, then all the traces $\tilde{\mathcal{A}}_{j}$ of $\mathcal{A}$ on its own hyperplanes are non-empty.

Consider now two hyperplanes $H_{a}$ and $H_{b}$. Note that, for all $j$ such that $1 \leq$ $j \leq m$, if the intersection $H_{j} \cap H_{a} \cap H_{b}$ is a proper affine subspace in $H_{a} \cap H_{b}$, then the projection $\operatorname{pr}_{a b}\left(N_{j}\right)$ of $N_{j}$ on the linear subspace subtending $H_{a} \cap H_{b}$ cannot vanish. Moreover the trace of the arrangement $\tilde{\mathcal{A}}_{a}$ on $H_{a} \cap H_{b}$ (as a hyperplane in $H_{a}$ ) equals

$$
\left\{\left(H_{j} \cap H_{a} \cap H_{b}, \frac{p r_{a b}\left(N_{j}\right)}{\left|p r_{a b}\left(N_{j}\right)\right|}\right)\right\},
$$

where $1 \leq j \leq m$ is such that $H_{j} \cap H_{a} \cap H_{b}$ is a proper subspace in $H_{a} \cap H_{b}$, so it coincides with the trace of $\tilde{\mathcal{A}}_{b}$ on the same $H_{a} \cap H_{b}$ (as a hyperplane in $H_{b}$ ). We say thus that the iterated trace of an arrangement $\mathcal{A}$ on several hyperplanes does not depend on the particular order in which these traces are taken.

Definition 6. A gliding d-arrangement of hyperplanes is a family

$$
\mathcal{A}=\left\{\left(H_{1}, v_{1}\right),\left(H_{2}, v_{2}\right), \ldots,\left(H_{m}, v_{m}\right)\right\}
$$

where each hyperplane $H_{j}$ is endowed with a normal velocity vector $v_{j}$. A pair $(H, v)$ as above will be called a gliding hyperplane.

Example 4. Let $P$ be a $d$-dimensional polytope in $\mathbb{E}^{d}$. Consider the gliding $d$ arrangement $\mathcal{A}_{P}$ consisting of all the hyperplanes supporting the facets of $P$ with the orientation given by the inner unit normal vectors $N_{j}$ and with velocity vectors $v_{j}=N_{j}$ for all $j$. We call $\mathcal{A}_{P}$ the gliding arrangement adapted to $P$.

A gliding arrangement $\mathcal{A}$ determines at each moment of time $t$ a $d$-arrangement

$$
\mathcal{A}(t)=\left\{H_{1}(t), H_{2}(t), \ldots, H_{m}(t)\right\}
$$


where $H_{j}(t)$ is the image of $H_{j}(0)=H_{j}$ under the translation by vector $t v_{j}$, i.e.

$$
H_{j}(t)=\left\{Q \in \mathbb{E}^{d} \mid \mathrm{d}\left(Q, H_{j}\right)-t\left\langle v_{j}, N_{j}\right\rangle=0\right\} .
$$

Note that the rank and the absolute rank of $\mathcal{A}(t)$ are constant. For a multi-index $\varepsilon \in\{ \pm 1\}^{m}$ denote the cell $C_{\varepsilon}$ by $C_{\varepsilon}(t)$ to indicate its dependence on the time parameter. Precisely,

$$
C_{\varepsilon}(t)=\left\{Q \in \mathbb{E}^{d} \mid \varepsilon_{j} \mathrm{~d}\left(Q, H_{j}(t)\right) \geq 0, \text { for all } j=1, \ldots, m\right\} .
$$

Note that if $C_{\varepsilon}\left(t_{0}\right)$ is bounded for some $t_{0}$, then it is bounded for all real $t$. Let

$$
W(t)=W_{\varepsilon}(t)=\operatorname{vol}_{d}(C(t))
$$

be its $d$-dimensional volume function.

Definition 7. A gliding $d$-arrangement $\mathcal{A}$ is of first type if it admits a bounded cell $C_{\varepsilon}\left(t_{0}\right)$ with non-zero $d$-dimensional volume, for some multi-index $\varepsilon$ and a real $t_{0}$.

Example 5. The gliding arrangement adapted to a polytope is of first type by definition.

Lemma 1. Let $\mathcal{A}$ be a gliding arrangement of hyperplanes in $\mathbb{E}^{d}$ of first type. Let $C(t)$ be a bounded cell of $\mathcal{A}$ of volume function $W$. Then $W$ is continuous and $\operatorname{supp} W$ is an interval. For all $t \notin \operatorname{supp} W, C(t)$ is empty.

Proof. The continuity of $W$ follows simply by induction on the dimension $d$, and we will skip its proof. If, for $i=0,1, t_{i} \in \mathbb{R}$ and $Q_{i} \in C\left(t_{i}\right)$, then for all $\lambda \in[0,1]$, the point $(1-\lambda) Q_{0}+\lambda Q_{1} \in C\left((1-\lambda) t_{0}+\lambda t_{1}\right)$. Thus if $t_{0} \neq t_{1}$ and if $\operatorname{vol}_{d}\left(C_{\varepsilon}\left(t_{1}\right)\right)>0$, then $\operatorname{vol}_{d}\left(C\left((1-\lambda) t_{0}+\lambda t_{1}\right)\right) \geq \lambda^{d} \operatorname{vol}_{d}\left(C\left(t_{1}\right)\right)>0$. Thus if $t_{0}<t_{1}$ are in supp $W$, then the whole interval $\left[t_{0}, t_{1}\right]$ is contained in supp $W$. By the same argument as above, if $t_{1} \in \operatorname{supp} W$ and $t_{0} \notin \operatorname{supp} W$ but $C\left(t_{0}\right)$ is non-empty, then $t_{0}$ is in the closure of $\operatorname{supp} W$, which is absurd. Thus $C(t)$ is empty for all $t \notin \operatorname{supp} W$.

Assume that $\left(H_{j}, v_{j}\right)$ and $\left(H_{0}, v_{0}\right)$ are gliding hyperplanes in $\mathbb{E}^{d}$ in general position.

Definition 8. We define the trace of the gliding hyperplane $H_{j}$ on $H_{0}$ to be the hyperplane $H_{j} \cap H_{0}$ endowed with the normal vector $\tilde{N}_{j}=\frac{p r_{0}\left(N_{j}\right)}{\left|p r_{0}\left(N_{j}\right)\right|}$ and with the velocity vector

$$
\tilde{v}_{j}=\left\langle v_{j}-v_{0}, N_{j}\right\rangle \frac{p r_{0}\left(N_{j}\right)}{\left|p r_{0}\left(N_{j}\right)\right|^{2}} .
$$

Note that in the orthogonal complement of $H_{0} \cap H_{j}$, the two bases $\left\{N_{0}, N_{j}\right\}$ and $\left\{\frac{p r_{j}\left(N_{0}\right)}{\left|p r_{j}\left(N_{0}\right)\right|^{2}}, \frac{p r_{0}\left(N_{j}\right)}{\left|p r_{0}\left(N_{j}\right)\right|^{2}}\right\}$ are dual to each other. The vector $\tilde{v}_{j}$ is exactly the $\frac{p r_{0}\left(N_{j}\right)}{\left|p r_{0}\left(N_{j}\right)\right|^{2}}$-component of the vector $v_{j}-v_{0}$.

Let $\mathcal{A}=\left\{\left(H_{1}, v_{1}\right),\left(H_{2}, v_{2}\right), \ldots,\left(H_{m}, v_{m}\right)\right\}$ be a gliding $d$-arrangement of hyperplanes, and let $\left(H_{0}, v_{0}\right)$ be a gliding hyperplane in $\mathbb{E}^{d}$, with $d \geq 2$.

Definition 9. We define the trace of the gliding d-arrangement $\mathcal{A}$ in $H_{0}$ to be the family $\tilde{\mathcal{A}}_{0}$ of all the traces of the gliding hyperplanes $\left(H_{j}, v_{j}\right)$ on $\left(H_{0}, v_{0}\right)$, where we choose only those hyperplanes $\left(H_{j}, v_{j}\right)$ such that $H_{j}$ and $H_{0}$ are in general position. 
Let $\mathcal{A}_{P}$ be the gliding arrangement adapted to some $d$-dimensional polytope $P$ in $\mathbb{E}^{d}$. Note that if $P$ is a $d$-dimensional rectangle, the traces of $\mathcal{A}_{P}$ on the hyperplanes $H_{j}$ containing the facets of $P$ are adapted to the respective facet $P \cap H_{j}$. Nevertheless, for a general polytope $P$ this is not necessarily true.

Lemma 2. Let $H_{0}$ be a gliding hyperplane, let $\mathcal{A}$ be a gliding arrangement of hyperplane in $\mathbb{E}^{d}$ with $d \geq 2$, and denote by $\tilde{\mathcal{A}}_{0}$ the trace of $\mathcal{A}$ on $H_{0}$. Then the arrangement $\tilde{\mathcal{A}}_{0}(t)$ is the trace of $\mathcal{A}(t)$ on $H_{0}(t)$, for all $t$.

In particular, we conclude that the iterated trace of a gliding arrangement $\mathcal{A}$ on several gliding hyperplanes does not depend on the particular order in which these traces are taken.

Proof. We can assume that $\mathcal{A}$ consists of a single gliding hyperplane $(H, v)$ and that the normal vectors $N$ and $N_{0}$ are linearly independent. We can identify $\mathbb{E}^{d}$ with the Euclidean real coordinate space $\mathbb{R}^{d}$ in such a way that $N_{0}=\mathbf{e}_{1}, N=$ $\frac{1}{\sqrt{b^{2}+c^{2}}}\left(b \mathbf{e}_{1}+c \mathbf{e}_{2}\right)$, with $c \neq 0, H_{0}(0)=\left\{x_{1}=0\right\}$ and $H(0)=\left\{b x_{1}+c x_{2}=0\right\}$.

Then $v_{0}=a \mathbf{e}_{1}$ and $v=\omega\left(b \mathbf{e}_{1}+c \mathbf{e}_{2}\right)$, for some real $\omega$; thus $H_{0}(t)=\left\{x_{1}=a t\right\}$ and $H(t)=\left\{b x_{1}+c x_{2}=\omega\left(b^{2}+c^{2}\right) t\right\}$ intersect at

$$
\left\{\left(a t, \frac{\omega\left(b^{2}+c^{2}\right)-a b}{c} t, x_{2}, \ldots, x_{d}\right) \mid t \in \mathbb{R}\right\} .
$$

Thus the trace $\widetilde{(H, v)_{0}}$ of the gliding hyperplane $(H, v)$ on $H_{0}$ has velocity vector $\frac{\omega\left(b^{2}+c^{2}\right)-a b}{c} \mathbf{e}_{2}=\left\langle v-v_{0}, N\right\rangle \frac{p r_{0}(N)}{\left|p r_{0}(N)\right|^{2}}$. Lemma 2 is thus proven.

Theorem 2. Let $\mathcal{A}$ be a gliding d-arrangement of hyperplanes in $\mathbb{E}^{d}$ of first type and of absolute rank $k$, and let $C$ be a bounded cell such that for some value $t_{0}$, $C\left(t_{0}\right)$ has non-zero d-volume. Then the function $W(t)=\operatorname{vol}_{d}(C(t))$ is a pluriphase degree $d$ Steiner-like function of class $\mathcal{C}^{k-1}$ on $\mathbb{R}$.

Proof. By induction on the dimension $d$.

The theorem is clear if $d$ is 1 . Note that in this case $W(t)$ is of class $\mathcal{C}^{1}$ only if the cell is non-empty for all $t$.

Assume the theorem has been proven for $(d-1)$-arrangements. The cell $C=C_{\varepsilon}$ of the $d$-arrangement $\mathcal{A}$ determines in the trace $\tilde{\mathcal{A}}_{j}$ a unique cell $C_{j}$ for each $j$. Note that $C_{j}(t)$ may be empty for some $t$ or even for all $t$. However, by the Lemma above, $C_{j}(t)$ is the $j$ th facet of $C(t)$, for all $t$ in supp $W$. Since $C$ is a bounded cell, the same is true for $C_{j}$. In particular we conclude that for all $j$ such that $C_{j}(t)$ has non-zero $d$-dimensional volume for some $t, \tilde{\mathcal{A}}_{j}$ is a $(d-1)$-arrangement of first type of absolute rank at least $k-1$. Note, however, the important fact that if $\operatorname{vol}_{d-1} C_{j}\left(t_{1}\right) \neq 0$ for at least one value $t_{1}$ for which $\operatorname{vol}_{d}\left(C\left(t_{1}\right)\right)=0$, then $\mathcal{A}$ must contain at least one hyperplane $H_{l}$, with $l \neq j$ such that the normal vectors $N_{l}$ and $N_{j}$ are linearly dependent. We conclude that $\mathcal{A}$ has absolute rank 1 in this case.

Denote the $(d-1)$-dimensional volume function of $C_{j}(t)$ by $W_{j}(t)$, for each $j$. Note also that the inner unit normal vector of the cell $C(t)$ along its facet $C_{j}(t)$ is $\varepsilon_{j} N_{j}$. By taking the one-sided derivatives with respect to $t$, we get

$$
W^{\prime}(t \pm)=-\chi(t \pm) \sum_{j=1}^{m}\left\langle v_{j}, \varepsilon_{j} N_{j}\right\rangle W_{j}(t \pm), \quad \text { for all } t
$$


where $\chi$ is the characteristic function of the interval supp $W$. The differentiation formula holds even if the sum has been restricted only to those terms for which $\tilde{\mathcal{A}}_{j}$ is of first type, and thus by the induction hypothesis we conclude that the function $W$ is a pluriphase degree $d$ Steiner-like function of class $\mathcal{C}^{k-1}$ on $[0,+\infty) \backslash \partial(\operatorname{supp} W(t))$.

If the absolute rank of $\mathcal{A}$ is 1 , the conclusion of the theorem is proven. Otherwise, the absolute rank of $\mathcal{A}$ is greater than 1 and, by the argument above, the volume function $W_{j}$ is null outside the interval $\operatorname{supp} W$ for all facet $j$. The theorem follows.

Corollary 3. Let $P$ be a d-dimensional polytope in $\mathbb{R}^{d}$. If the family $\mathcal{N}=$ $\left\{N_{1}, N_{2}, \cdots, N_{m}\right\}$ of the inner normal vectors of the facets of $P$ has absolute rank $k$, then the inner neighborhood volume function $V_{P}(r)$ is a pluriphase degree $d$ Steiner-like function of class $\mathcal{C}^{k-1}$ on $[0,+\infty)$.

Proof. Consider the gliding $d$-arrangement $\mathcal{A}_{P}$ adapted to $P$. The polytope $P$ is the cell $C=C_{(+1,+1, \ldots,+1)}$. We first show that for non-negative $t, C(t)=$ $C_{(+1,+1, \ldots,+1)}(t)$ coincides with the $t$-interior of $P$, i.e.

$$
P(t)=\{Q \in P \mid \mathrm{d}(Q, \partial P) \geq t\} .
$$

Indeed, if $Q \in C(t)$, then for all $j, \mathrm{~d}\left(Q, H_{j}(t)\right) \geq 0$. So d $\left(Q, P \cap H_{j}\right) \geq \mathrm{d}\left(Q, H_{j}\right) \geq t$, for all facets $P \cap H_{j}$ of $P$. Conversely, if $Q \in P$ is a point satisfying $\mathrm{d}(Q, \partial P) \geq t$, then $\mathrm{d}\left(Q, \overline{\mathbb{R}^{d} \backslash P}\right) \geq t$. Since any hyperplane $H_{j}(0)$ supporting a facet of $P$ is contained in the complement $\overline{\mathbb{R}^{d} \backslash P}$, we conclude that $\mathrm{d}\left(Q, H_{j}(0)\right) \geq t$ for all $j$. Thus $Q \in C(t)$. The proof follows now by directly applying the theorem to the gliding $d$-arrangement $\mathcal{A}_{P}$ and to the cell $P$ and the relation $W(r)=\operatorname{vol}_{d}(P)-$ $V_{P}(r)$, valid for all positive $r$.

Moreover, the theorem below proves that, for each $0 \leq k \leq d$, there exists a polytope $P$ such that the absolute rank of $\mathcal{N}$ is $k$ but $\bar{V}_{P}(r)$ is not of class $\mathcal{C}^{k}$ on $[0,+\infty)$. So, in this sense, the bound for the differentiability class given in Corollary 3 is the best possible result.

Theorem 4. Let $1 \leq k \leq s \leq d$. Then there exists a polytope in $\mathbb{R}^{d}$ whose family $\mathcal{N}$ of inner normal vectors of facets has absolute rank $k$ and whose inner neighborhood volume function $V_{P}$ is of differentiability class $\mathcal{C}^{(s-1)}$, but not $\mathcal{C}^{s}$.

Proof. Consider the following construction, starting with be a polytope $P$ in $\mathbb{R}^{d}$ having $m$ facets, having inradius $g$, and absolute rank $k$ of the family $\mathcal{N}$ of normal vectors. We define the roof of $P$ to be the polytope $\Gamma(P)$ in $R^{d+1}$ given by

$$
\Gamma(P)=\left\{x^{\prime}=\left(x, x_{d+1}\right) \mid 0 \leq x_{d+1} \leq g, x \in P\left(x_{d+1}\right)\right\} .
$$

If, for all non-negative $r$, we canonically identify $\mathbb{R}^{d}$ with $\left\{(x, r) \mid x \in \mathbb{R}^{d}\right\}$, the $r$ interior of $P$ is congruent with the horizontal slice $\left\{x_{d+1}=r\right\}$ in $\Gamma(P)$, so $\Gamma(P)$ is the graph of the shrinking polytope $P_{r}$, as $r$ varies in $[0,+\infty)$ (see Figure 3 ).

Note that $\Gamma(P)$ is a polytope with $m+1$ facets and of absolute rank $k+1$. We identify $P$ with the facet $P \times\{0\}$ of $\Gamma(P)$, whose inner unit normal vector is the basis vector $\mathbf{e}_{d+1}$. Note that the outer dihedral angle between $P$ and any other facet of $\Gamma(P)$ equals $\frac{3 \pi}{4}$. We conclude that, for all $r$, the translation of vector $r \mathbf{e}_{d+1}$ in $R^{d+1}$ maps $\Gamma(P((1+\sqrt{2}) r))$ onto $\Gamma(P)(r)$; thus the inradius of $\Gamma(P)$ is $\frac{g}{1+\sqrt{2}}$. 


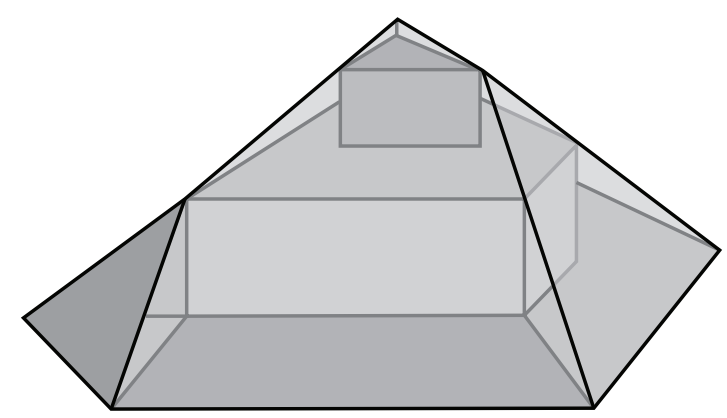

Figure 3. The roof of the polygon $A B C D E$ shown in Figure 2.

As the volume of $\Gamma(P)$ is given by

we obtain that

$$
\operatorname{vol}_{d+1}(\Gamma(P))=\int_{0}^{\infty} \operatorname{vol}_{d}(P(\rho)) d \rho
$$

$$
\begin{aligned}
\operatorname{vol}_{d+1}(\Gamma(P)(r)) & =\operatorname{vol}_{d+1}(\Gamma(P((1+\sqrt{2}) r))) \\
& =\int_{0}^{\infty} \operatorname{vol}_{d}(P((1+\sqrt{2}) r+\rho)) d \rho \\
& =\int_{(1+\sqrt{2}) r}^{\infty} \operatorname{vol}_{d}(P(\rho)) d \rho
\end{aligned}
$$

or, equivalently,

$$
W_{\Gamma(P)}^{\prime}(r)=-(1+\sqrt{2}) W_{P}((1+\sqrt{2}) r), \text { for all } r \in[0,+\infty)
$$

Coming back to the proof of the theorem, let $d^{\prime}=d-k+1$ and $m=s-k+1$ and consider a sequence $0<a_{1}=a_{2}=\cdots=a_{m}<a_{m+1} \leq \ldots a_{d^{\prime}}$. Let $R$ be the rectangle $R_{a_{1}, a_{2}, \ldots, a_{d^{\prime}}}$ in $\mathbb{R}^{d^{\prime}}$ as in the example considered in the first section and let $P=\Gamma^{k-1}(R)=\Gamma(\Gamma(\ldots(R) \ldots))$. Then $P$ is a polytope in $\mathbb{R}^{d}$ of absolute rank $k$ and its volume function $W_{P}$ is of class $\mathcal{C}^{(s-1)}$, but not $\mathcal{C}^{s}$.

\section{ACKNOWLEDGEMENTS}

The authors cordially thank Ali Deniz and Yunus Ozdemir from Anadolu University for drawing the figures.

\section{REFERENCES}

[1] M. Berger, Geometry, Springer-Verlag, Berlin, 1987. MR0882541(88a:51001a) MR0882916 (88a:51001b)

[2] J.-M. Morvan, Generalized Curvatures, Springer-Verlag, Berlin, 2008. MR2428231 (2009m:53205)

[3] H. Federer, Curvature measures, Trans. Amer. Math. Soc. 93 (1959), 418-491. MR0110078 $(22: 961)$

[4] A. Gray, Tubes, Birkhäuser, Basel, 2004. MR2024928 (2004j:53001)

[5] R. Schneider, Convex Bodies: The Brunn-Minkowski Theory, Cambridge University Press, Cambridge, 1993. MR 1216521 (94d:52007)

[6] M. L. Lapidus and M. van Frankenhuijsen, Fractal Geometry, Complex Dimensions and Zeta Functions, Springer-Verlag, Berlin, 2006. MR2245559 (2007j:11001) 
[7] M. L. Lapidus and E. P. J. Pearse, Tube formulas and complex dimensions of self-similar tilings, Acta Mathematicae Applicandae, 112, No. 1 (2010), 91-137. MR2684976

[8] M. L. Lapidus and E. P. J. Pearse, Tube formulas for self-similar fractals, in Analysis on Graphs and Its Applications (P. Exner, J. P. Keating, C. Bristol, P. Kuchment, T. Sunada, and A. Teplyaev, eds.), Proc. Sympos. Pure Math., vol. 77, Amer. Math. Soc., Providence, RI, 2008, pp. 211-230. MR2459871 (2010a:28012)

[9] M. L. Lapidus and E. P. J. Pearse, and S. Winter, Pointwise tube formulas for fractal sprays and self-similar tilings with arbitrary generators, Adv. Math. 227 (2011), 1349-1398.

[10] A. Deniz, S. Kocak, Y. Ozdemir, and A. E. Ureyen, Tube formulas for self-similar fractals with non-Steiner-like generators, arXiv:0911.4966.

Department of Mathematics, Anadolu University, 26470 Eskişehir, Turkey

E-mail address: skocak@anadolu.edu.tr

Department of Mathematics and Statistics, University of Melbourne, Parkville, Melbourne, ViC 3010, Australia

E-mail address: aratiu@unimelb.edu.au 